\title{
Delta-Centralization Fails to Control for Population Stratification in Genetic Association Studies
}

\author{
Tony Dadd ${ }^{a}$ b Cathryn M. Lewis ${ }^{b, c}$ Michael E. Weale ${ }^{b}$ \\ a Unilever Statistics Group, Colworth Science Park, Sharnbrook, Bedford, ${ }^{\mathrm{b}}$ Department of Medical and Molecular \\ Genetics and 'MRC SGDP Centre, Institute of Psychiatry, King's College London, London, United Kingdom
}

\section{Key Words}

Population stratification - Delta-centralization - Genomic control $\cdot$ Simulation $\cdot$ Allele frequency matching

\begin{abstract}
Objective: To investigate the validity of simulations and assumptions used to underpin the delta-centralization (DC) method for correcting for population stratification in genetic association studies; to assess the effectiveness of DC compared to genomic control (GC) under valid simulation conditions; and to highlight other studies employing similarly flawed simulations. Methods: DC and GC use data from unlinked null loci to correct the test statistic at the target locus, but differ in the way the correction is performed. We compare DC and GC under two simulation approaches: an invalid approach adopted by the originators of DC, which permits subpopulation allele frequency matching of null markers to the target locus; and a valid approach, based on the Balding-Nichols model, which does not allow subpopulation matching. Results: DC works under invalid simulation conditions (subpopulation allele frequency matching), but not under our valid ones. We use theoretical arguments to assert that there are no valid conditions under which DC
\end{abstract}

might work. We identify several studies which have adopted similarly invalid simulation approaches in this field. Conclusion: DC fails to control for population stratification and should not be used. Other results from studies which have used the same invalid simulation approach should be treated cautiously.

Copyright $\odot 2010$ S. Karger AG, Basel

\section{Introduction}

The number of genetic association studies using unrelated individuals has exploded in recent years. These studies are prone to confounding from imbalances in sampling cases and controls from different underlying subpopulations, or from the inclusion of admixed individuals whose genetic ancestry is correlated with disease status. This leads to allele frequency differences between cases and controls which are unrelated to disease status, and can give rise to both false positive and false negative signals of association [1]. We define population stratification (PS) as occurring when the average degree of sharing of genetic ancestry among some individuals (e.g. sampled within a subpopulation) is greater than among other in-

\section{KARGER}

Fax +41613061234

E-Mail karger@karger.ch

www.karger.com
(C) 2010 S. Karger AG, Basel

0001-5652/10/0694-0285\$26.00/0

Accessible online at:

www.karger.com/hhe
Mike Weale, $\mathrm{PhD}$

Department of Medical \& Molecular Genetics

King's College London, 8th Floor, Tower Wing, Guy's Hospital

London SE1 9RT (United Kingdom)

Tel. +44 (0)20 7188 2601, Fax +44 (0)20 7188 2585, E-Mail michael.weale@ kcl.ac.uk 
dividuals (e.g. sampled between subpopulations). For convenience and for consistency with the approaches adopted by other researchers, we will adopt a discrete subpopulation model for describing PS. However, we agree with Astle and Balding [20] that, for human beings in particular, there are no uniform barriers to gene flow that would result in a rigid block-like structure in the pairwise matrix of genetic ancestry in a sample of individuals.

Many statistical methods have been proposed to correct for PS, all based on the principle of using a set of 'null' markers to adjust the test statistic at the locus of interest. Here we define the null marker set to be a set of unlinked markers, in linkage equilibrium within any given subpopulation, presumed to be neutral with respect to the phenotype in question, and typically made up of bi-allelic single nucleotide polymorphism (SNP) markers. Some methods do allow some SNPs in the null marker set to be in linkage disequilibrium, but to work effectively all these methods require a large degree of linkage equilibrium among null SNPs. Statistical methods that use a null marker set include genomic control (GC) [2], structured association [3], principal components analysis [4], stepwise/penalised logistic regression [5] and, recently, delta-centralization (DC) $[6,7]$. GC corrects for false positive signals of association only. In principle, all the other methods can also correct for false negative signals, in effect by recognising and correcting for situations where PS leads to an allele frequency difference between cases and controls in one direction and causal association leads to an allele frequency difference in the opposite direction. However, GC enjoys some other advantages. It is easy to implement and requires only a few null markers to operate efficiently [8]. It can also operate effectively under conditions of extreme case-control imbalance (e.g. all cases sampled from one subpopulation, all controls from another), a scenario which may have been encouraged by the recent advent of 'standard' control datasets such as the WTCCC reference panels [9]. In contrast, covariate-based methods such as structured association, principal components analysis and logistic regression will fail under this scenario, once enough null markers have been typed to properly characterise the underlying population structure.

GC methods assume that the typical $\chi^{2}$ test statistic for association is distributed as an inflated central $\chi^{2}$ distribution under the null hypothesis with PS, and estimate the inflation factor $\lambda$ via some summary statistic derived from the genotyped set of unlinked null SNPs. In contrast, Gorroochurn et al. [6, 7] champion the case for a non-central $\chi^{2}$ distribution for the test statistic and propose an alternative DC method which corrects the test statistic under this assumption. The DC method has not been used in any association studies to our knowledge, but is has inspired a number of subsequent theoretical and simulation-based studies [11, 17-19].

Here we show that DC depends on the assumption that null markers can be chosen in such a way that the withinsubpopulation allele frequencies of these markers will to some extent match the within-subpopulation allele frequencies of the target locus of interest. The originators of the DC method $[6,7]$ noted that it is not possible for the investigator to perform this matching directly, because the subpopulation allele frequencies are unknown. However, these authors, and also some other authors [11, 1719], have implicitly assumed that it is possible to perform this matching indirectly, under the following conditions. First, assume that there exists some population genetic mechanism under which a target locus and an identifiable set of other markers tend to evolve towards the same set of subpopulation allele frequencies, albeit with some degree of error allowed. Secondly, allow the investigator to select null markers according to some matching criterion applied at the whole-population level, such as those markers with an observed minor allele frequency within $\pm 5 \%$ of that of the target locus in the control group. Simulations based on these conditions will result in subpopulation allele frequency matching.

In this paper we argue that the first condition described above is impossible, except under very special and unusual conditions considered in the Discussion. This is due to the directionless quality of genetic drift, which ensures that the direction of drift of allele frequencies at one locus cannot influence the direction of drift at other unlinked loci. This then invalidates all of the simulations performed by Gorroochurn et al. [6, 7], as well as some simulations performed by other authors which we describe later. When appropriate population genetic simulations are performed, DC fails whereas GC performs well. DC should therefore be dropped from the list of valid methods for the correction of PS in association studies.

\section{Delta-Centralization}

DC attempts to estimate a non-centrality parameter for the test SNP directly from a panel of $L$ null SNPs, and then uses that estimate in the testing procedure. The derivation of DC is given in Gorroochurn et al. [6] and is briefly repeated here for the case of the dominant genetic model. Gorroochurn et al. [6] also present a similar deri- 
vation for the additive genetic model, using the CochranArmitage test for trend. Zang et al. [11] extended the method to consider robust tests under uncertain genetic models.

Consider a study where a SNP genotype is tested against case-control status for association under a dominant genetic model (table 1). Gorroochurn et al. [6] define the frequency of the test SNP genotype in the cases and controls as $s$ and $t$ respectively, where:

$$
s=a_{0} / m \quad t=c_{0} / n
$$

Then the usual test for difference in the proportions of cases and controls with the test SNP genotype is given by a $Z$ statistic:

$$
Z=\frac{(s-t)}{\sqrt{\frac{s(1-s)}{m}+\frac{t(1-t)}{n}}}
$$

Under the null hypothesis of no difference between cases and controls and no PS, $Z$ is asymptotically distributed $\mathrm{N}(0,1)$ and thus $Z^{2}$ is distributed $\chi_{1}^{2}$. It can be shown that $Z^{2}$ is the standard Pearson $\chi^{2}$ statistic for association for a $2 \times 2$ table. Under the alternate hypothesis of $s \neq t, Z$ is asymptotically distributed $\mathrm{N}(\delta, 1)$ and thus $Z^{2}$ is distributed as a non-central $\chi^{2}, \chi_{1}^{2}\left(\delta^{2}\right)$, where $\delta^{2}$ is the non-centrality parameter. Gorroochurn et al. [6] also refer to $\delta^{2}$ as $\tau$ (not to be confused with the $\tau$ used in Devlin et al. [10]).

There are two ways that one can obtain $s \neq t$ (and thus $\delta^{2}>0$ ), one is if there is a genuine causal relationship at the test SNP, and the other is under PS with different allele frequencies between subpopulations and unbalanced sampling of cases and controls from these subpopulations. Gorroochurn et al. [6] seek to estimate that part of the non-centrality parameter due just to PS by estimating $\delta$ in the set of $L$ null SNPs. They then subtract that part out of the test statistic for the test SNP. Thus Gorroochurn et al. [6] define their inflation-corrected test statistic as:

$$
T_{D C}=\left\{\operatorname{sign}\left(a_{0} d_{0}-b_{0} c_{0}\right) \times \sqrt{X^{2}}-\delta\right\}^{2} \sim \chi_{1}^{2}(0)
$$

The sign correction is necessary to ensure that we are on the 'correct' side of the $\mathrm{N}(\delta, 1)$ distribution when $\delta$ is subtracted, with $a_{0} d_{0}-b_{0} c_{0}$ being positive when $s>t$. Note that it is possible for $T_{D C}$ to increase in magnitude after DC correction, when $s>t$ and $\delta$ is negative or when $s<t$ and $\delta$ is positive. This occurs when a real causal signal and a false PS signal act in opposite directions, and cor-
Table 1. Notation used for deriving DC for SNP association of genotype $\mathrm{M}$ with case-control status

\begin{tabular}{llll}
\hline & $\mathrm{M}$ & $\mathrm{M}^{\prime}$ & Total \\
\hline Cases & $a_{0}$ & $b_{0}$ & $m$ \\
Controls & $c_{0}$ & $d_{0}$ & $n$ \\
Total & $a_{0}+c_{0}$ & $b_{0}+d_{0}$ & $N$ \\
\hline
\end{tabular}

responds to a situation where a false negative result is being corrected.

Two alternative methods are used to estimate $\delta$ from a set of unlinked null SNPs. In Gorroochurn et al. [6], estimators for $s$ and $t$ are obtained from combining information from $L$ null SNPs:

$$
\hat{s}=\frac{1}{m L} \sum_{i=1}^{L} a_{i}, \quad \hat{t}=\frac{1}{n L} \sum_{i=1}^{L} c_{i}
$$

These estimators are then combined to form an estimate for $\delta$ :

$$
\hat{\delta}_{2006}=\frac{\hat{s}-\hat{t}}{\sqrt{\frac{\hat{s}(1-\hat{s})}{m}-\frac{\hat{t}(1-\hat{t})}{n}}}
$$

In Gorroochurn et al. [7], estimates for $\delta$ are obtained from each of the individual null SNPs:

$$
\begin{aligned}
& \hat{\delta}_{i}=\frac{\hat{s}_{i}-\hat{t}_{i}}{\sqrt{\frac{\hat{s}_{i}\left(1-\hat{s}_{i}\right)}{m_{i}}-\frac{\hat{t}_{i}\left(1-\hat{t}_{i}\right)}{n_{i}}}} \\
& s_{i}=a_{i} / m_{i}, \quad t_{i}=c_{i} / n_{i}
\end{aligned}
$$

These estimators are then averaged to form an overall estimate for $\delta$ :

$$
\hat{\delta}_{2007}=\frac{1}{L} \sum_{i=1}^{L} \hat{\delta}_{i}
$$

The difference between $\hat{\delta}_{2006}$ and $\hat{\delta}_{2007}$ is thus the difference between taking a ratio of averages versus taking an average of ratios.

For maximising efficiency in reducing both the genotyping costs and the bias in estimating $\delta$, Gorroochurn et al. $[6,7]$ suggest that the null SNPs should be matched reasonably closely to the test SNP on genotype or allele frequency in the controls. Their guidelines are for \pm 0.1 to \pm 0.15 on SNP genotype or allele frequency in the controls. 


\section{Genomic Control}

GC methods assume that the usual test statistic for association of a SNP of interest (the 'test' SNP) is inflated to become distributed as $\lambda \chi_{1}^{2}$ under the null hypothesis with PS. Lambda is estimated from the median or mean $\chi^{2}$ statistic $\left(\chi_{\text {median }}^{2}\right.$ or $\left.\chi_{\text {mean }}^{2}\right)$ from a set of $L$ null SNPs. $\chi_{\text {median }}^{2} / 0.4549$ and $\chi_{\text {mean }}^{2}$ are both consistent estimators of $\lambda$, and so if one ignores the estimation uncertainty then division of the test statistic by these estimators of $\lambda$ returns its distribution under the null to $\chi_{1}^{2}$. This gives rise to the $\mathrm{GC}_{\text {med }}$ method if $\lambda=\chi_{\text {median }}^{2} / 0.4549$ is used [2], and to the $\mathrm{GC}_{\mathrm{mu}}$ method if $\lambda=\chi_{\text {mean }}^{2}$ is used [12]. An $F_{1, \mathrm{~L}}$ distribution is obtained if estimation uncertainty is considered and $\lambda=\chi_{\text {mean }}^{2}$ is used, and this gives rise to the GCF method [13]. See Dadd et al. [8] for further details and references.

\section{Distributional Theory}

Both GC and DC and be linked through the same distributional theory. For example, Devlin et al. [10] considered the case of the allelic $\chi^{2}$ test for association (formed from the $2 \times 2$ table of allele counts for a diallelic marker in cases and controls). They showed that the distribution of the $\chi^{2}$ statistic for a given test SNP, T, under the null with PS, is an inflated non-central $\chi^{2}, \tau^{2} \chi_{1}^{2}\left(\delta_{T}^{2}\right)$. The inflation factor $\tau^{2}$ is due to the correlation of allele states within individuals (since alleles are sampled within subpopulations rather than across all subpopulations), and this inflation is not expected to be present for tests based on genotype counts such as the Cochran-Armitage trend test [14]. The non-centrality parameter is the square of the bias parameter

$$
\delta_{T}=\sum_{j=1}^{K} p_{T j} f_{j}
$$

for a PS model of $K$ discrete subpopulations where $p_{T j}$ is the frequency of the test SNP of interest in subpopulation $j$ and $f_{j}$ is the difference in the proportion of cases and controls sampled from subpopulation $j$.

The key difference is that DC assumes that $\delta_{T}$ is directly estimable from a set of unlinked null markers, whereas GC takes the view that it is not. This difference is directly related to the assumption of subpopulation allele frequency matching (reviewed in the next section), as without this assumption it is not possible for an unlinked set of markers to correctly gauge the sign of $\delta_{T}$, and this sign correction is required in order for DC to work (see previous section). Devlin et al. [10], in contrast, take the view that under a broad range of population genetic models it is reasonable to assume that $\mathrm{E}\left[\delta_{i}\right]=0$ over any set of null SNPs $(i=1, \ldots, L)$, thus making the signed value of $\delta_{T}$ inestimable from other null markers. Devlin et al. [10] also show that if PS is characterised by Wright's population structure coefficient $F_{S T}$, then

$$
\operatorname{Var}\left[\delta_{i}\right]=F_{S T} p_{i}\left(1-p_{i}\right) \sum_{j=1}^{K} f_{j}, \quad \Omega
$$

where $p_{i}$ is the average frequency of SNP $i$ over all subpopulations. Finally, they show that if one further assumes that $\delta_{i}$ is normally distributed among null SNPs, then it follows that the distribution of allelic $\chi^{2}$ statistics under the null with PS, where each statistic is drawn from $\tau^{2} \chi_{1}^{2}\left(\delta_{i}^{2}\right)$ with $\delta_{i} \sim \mathrm{N}\left(0, \operatorname{Var}\left[\delta_{i}\right]\right)$, becomes an inflated central $\chi^{2}\left(\tau^{2}+\eta^{2}\right) \chi_{1}^{2}$, where

$$
\eta^{2}=\frac{n F_{S T}}{4} \sum_{j=1}^{K} f_{j}
$$

and where $n$ is the number of controls in a 1:1 case-control study. Usefully, the inflation factor $\lambda=\left(\tau^{2}+\eta^{2}\right)$ does not depend on $p_{i}$, so in principle one does not need to match the allele frequency of null SNPs to the test SNP when employing an allelic $\chi^{2}$ test. Zheng et al. [17] establish that this property is also true for the Cochran-Armitage trend test, but not for the dominant/recessive genotypic tests.

A point of theoretical interest arises from the fact that all the SNPs considered here (both test and null) are genotyped in the same individuals. This means that the number of cases and controls drawn from each subpopulation, although unknown, is constant across SNPs. This allows Devlin et al. [10] to treat

$$
\sum_{j=1}^{K} f_{j}
$$

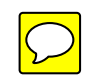

as a constant in deriving their results. It also means that there is a small deflation in the null distribution of the $\chi^{2}$ test statistic, relative to the variance used in the standard formula, since the latter assumes in effect that each SNP is genotyped in a separate sample of individuals drawn from a common multinomial distribution across all subpopulations. However, this deflation is generally small for plausible values of $F_{S T}$.

The preceding exposition highlights that the key difference between GC and DC is in the way the test statistic is conditioned. The distribution moves from a non-central $\chi^{2}$ when conditioned on known $\delta_{T}$ in DC, to an inflated central $\chi^{2}$ when conditioned on unknown $\delta_{T}$ but 
which is drawn from a known distribution $\delta_{i} \sim \mathrm{N}(0$, $\left.\operatorname{Var}\left[\delta_{i}\right]\right)$ in GC. Devlin et al. [10] justify the assumption of normality by reference to the central limit theorem in cases where the number of subpopulations, $K$, is large. For cases where $K$ is small, say $K=2$, departures from normality are expected and thus one cannot guarantee a perfect inflated central $\chi^{2}$. This motivates an empirical comparison of GC and DC for the case of $K=2$, which we perform next.

\section{Simulation Techniques}

Gorroochurn et al. $[6,7]$ model SNPs with specified allele frequencies in $m$ cases and $n$ controls from $K$ underlying the subpopulations $(i=1,2, \ldots, K)$ based on parameters $\pi_{i}, d_{i}$, and $r_{i}$.

$\pi_{i}$ are the relative sizes of the subpopulations, where by definition:

$$
\sum_{i=1}^{K} \pi_{i}=1
$$

$d_{i}$ is the probability of disease (D), given that the individual is in the subpopulation $i$.

$r_{i}$ is the probability of having SNP genotype M (i.e. one or more copies of the risk allele), given that the individual is in the subpopulation $i$.

$s, t$ are the probabilities of having SNP genotype M given case/control status.

Assuming that disease status is independent of SNP genotype in each subpopulation, then:

$$
P(D \cap M)=P(D) P(M)=d_{i} r_{\mathrm{i}}
$$

Conditional probability gives:

$$
s=P(M \mid D)=\frac{P(D \cap M)}{P(D)}
$$

Scaling by the relative sizes of the subpopulations and adding over all subpopulations gives $s$ :

$$
s=\frac{\sum_{i=1}^{K} \pi_{i} d_{i} r_{i}}{\sum_{i=1}^{K} \pi_{i} d_{i}}
$$

Using a similar argument for the controls (who are unaffected) gives $t$ :

$$
t=\frac{\sum_{i=1}^{K} \pi_{i}\left(1-d_{i}\right) r_{i}}{\sum_{i=1}^{K} \pi_{i}\left(1-d_{i}\right)}
$$

Evaluation of Delta-Centralization
The main simulation technique used by Gorroochurn et al. $[6,7]$ involves first specifying particular combinations of $\pi_{i}, d_{i}$, and $r_{i}$ in order to induce a range of theoretical values of $\delta$, and then calculating $s$ and $t$ from the equations given directly above. These parameters are then fixed for both the test SNP and the null SNPs, resulting in what we here term exact subpopulation allele frequency matching.

Binomial sampling from $\operatorname{Bin}(m, s)$ for cases and from $\operatorname{Bin}(n, t)$ for controls is used to obtain genotype counts for all SNPs. This binomial sampling scheme in effect assumes that each null SNP is genotyped in a separate sample of individuals, as this allows the counts of each genotype for cases and controls in each subpopulation to be multinomially distributed and thus for their sum over subpopulations to be binomially distributed. As we discuss in the previous section, the fact that the same individuals are used in genotyping each SNP results in slightly less variance in $\hat{s}$ and $\hat{t}$, but this effect is not great. For consistency with the results of Gorroochurn et al. [6,7], we employ the same binomial sampling scheme in our simulations.

In some of their simulations, Gorroochurn et al. [6, 7] allow for some additional variation in true subpopulation allele frequencies between the test SNP and the null SNPs. However, it is still assumed that null SNPs can be selected such that their expected subpopulation allele frequencies equal those of the test SNP (we refer to this as inexact subpopulation allele frequency matching). In Gorroochurn et al. [6] the allele frequencies are uniformly distributed, while in Gorroochurn et al. [7] a beta distribution is used (not to be confused with the beta distribution used in the Balding-Nichols model). In both methods the allowed variation is centred about a subpopulation-specific mean. Therefore, even when random errors are added, these simulations still contain the key assumption that subpopulation allele frequency matching is possible.

The subpopulation allele frequency matching simulation approach of Gorroochurn et al. [6, 7], and of other authors listed in the next section, does not reflect any process that can reasonably be undertaken by the researcher, because subpopulations are unknown. While there are methods which allow underlying structure to be inferred (see for example ref [3]), these rely on having genotype data over a much larger set of null SNPs than are proposed for DC. Nor can subpopulation allele frequency matching be expected to arise from any reasonable form of genetic drift under a neutral selection model, for reasons illustrated in figure 1. It is not possible for the direc-

Hum Hered 2010;69:285-294 


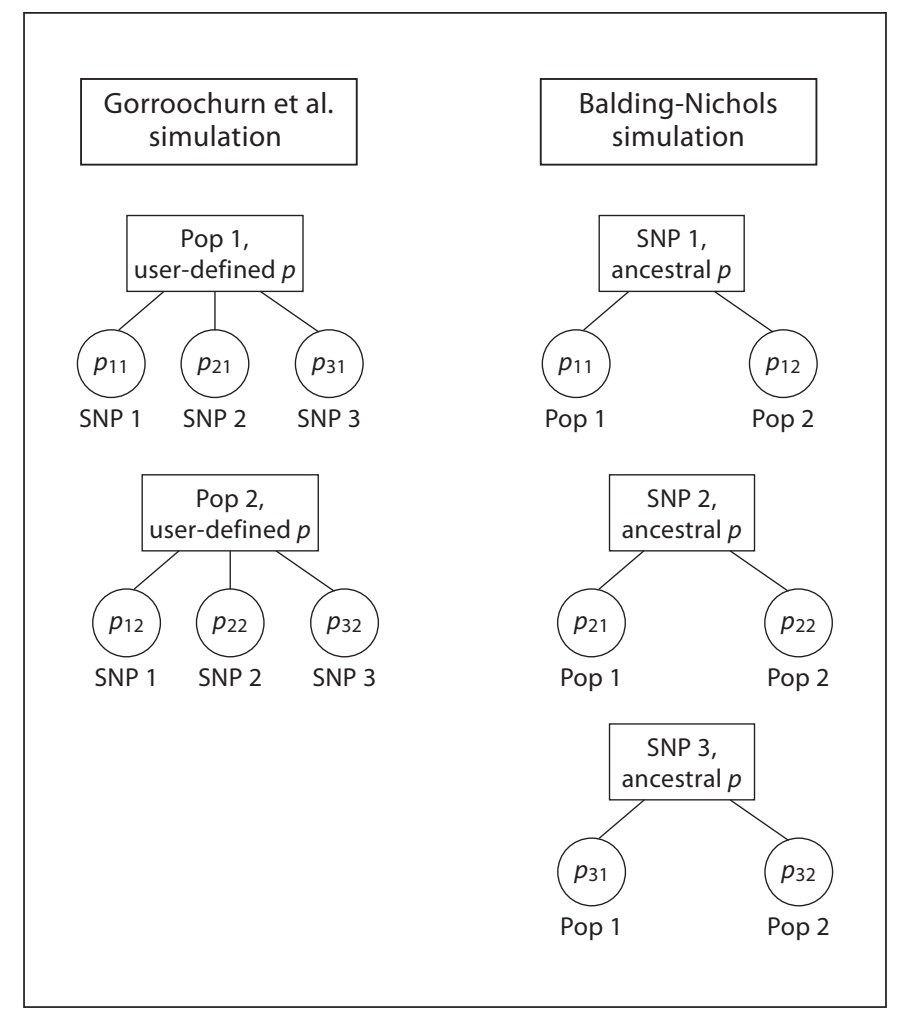

Fig. 1. Difference between Gorroochurn et al's simulation approach and a Balding-Nichols approach, illustrated using 3 SNPs drawn from 2 subpopulations. In Gorroochurn et al, mean allele frequencies $p$ are fixed in advance for each subpopulation. These are then resolved into a specific value $\mathrm{p}_{\mathrm{ij}}$ for SNP $i$ in Pop $j$ by the addition of a random error term (although this error is zero in some of their simulations). In the Balding-Nichols approach, mean allele frequencies are fixed in advance for each SNP. These mean values can be interpreted as the ancestral allele frequencies in a population-splitting model. These are then resolved into a specific value $\mathrm{p}_{\mathrm{ij}}$ for SNP $i$ in Pop $j$ by the addition of a random error term derived from the beta distribution. The independent simulations for each SNP reflect the property that the direction of genetic drift is independent and random among unlinked SNPs.

tion of genetic drift in neutral unlinked loci to be predicted based on the direction of drift in a target locus, which is what subpopulation allele frequency matching requires. For example, a widely used population genetic model is the Balding-Nichols model, which models the drift in allele frequencies as a beta distribution (about a global mean for each SNP, not a subpopulation-specific mean) [15]. The direction of genetic drift in each subpopulation is random under this model, as with any reasonable neutral population genetic model. Thus while the allele frequency of the test SNP might drift up in one sub- population and down in another, the opposite might happen for a separate unlinked null SNP under the same conditions. If the amount of drift is the same for both SNPs in both subpopulations, their overall allele frequencies in the association study will remain roughly constant, but with very different frequencies in the subpopulations. Thus matching SNPs based on overall frequencies will not remove the problem, as matching of allele frequencies within each subpopulation will still not be possible. In our simulation studies below, we contrast the results of Gorroochurn et al.'s simulation strategy with those obtained from a Balding-Nichols population genetic model.

\section{Simulations in Other Studies of PS Control}

We have identified similar simulation approaches employing subpopulation allele frequency matching in a number of papers by Zheng and colleagues (Zheng et al. [17, 18], Zang et al. [11], Zhang et al. [19]). We note that the term 'allele frequency matching' has many different meanings, both within these papers and in the literature more generally, and care is needed in distinguishing these. The term can be used to refer to either populationlevel matching (where overall allele frequencies across all subpopulations are matched) or to subpopulation matching (where allele frequencies within subpopulations are matched). It can be used to refer to exact matching (where the true subpopulation allele frequencies are the same, although some sampling variation in observed genotype frequencies is allowed) or inexact matching (where the true subpopulation allele frequencies share the same subpopulation-specific mean, but with some variation about the mean allowed). Finally, it can be used to either refer to a selection process undertaken by the researcher, or to a process implicitly assumed to arise as the result of some population genetic process.

Because they do not reflect any reasonable natural or artificial process, we designate as 'flawed' all simulations which employ subpopulation allele frequency matching, whether exact or inexact and whether assumed to arise by researcher selection or by a population genetic process.

Zheng et al. [17] investigated the effect of the underlying genetic model on GC. They employed two simulation approaches, the first of which allowed invalid exact subpopulation allele frequency matching of all target and null loci. The second used a uniform distribution for null locus subpopulation allele frequencies about a popula- 
tion-level mean, and so did not result in subpopulation allele frequency matching. Zheng et al. [16] investigated robust GC methods, and here they used what we term inexact and valid population-level (rather than subpopulation level) allele frequency matching simulations. Zang et al. [11], in a study comparing robust methods for GC and DC, presented two types of simulations, the first resulting in invalid inexact subpopulation allele frequency matching (their 'algorithm (i)' or 'PS' algorithm) and the second resulting in valid inexact population-level allele frequency matching (their 'algorithm (ii)' or 'CR' algorithm). Zheng et al. [18] developed and compared new methods inspired by GC and DC, including a hybrid GC+DC approach. They also used two types of simulation, here resulting in exact and inexact subpopulation allele frequency matching respectively, both of which are invalid. The sampling distribution for the inexact subpopulation matching was derived from empirical HapMap data, but it is important to note that the assumption of subpopulation allele frequency matching was something imposed by the authors and did not arise naturally from the HapMap data itself.

Finally, Zhang et al. [19] compared GC and DC against a hybrid GC+DC approach. Three different simulation types were used - 'random SNPs', 'differential SNPs' and 'stratified sampling.' 'Random SNPs' and 'stratified sampling' employed a beta distribution to sample subpopulation allele frequencies from a common 'ancestral' frequency, whereas 'differential SNPs' imposed pre-determined subpopulation allele frequencies for the target SNP. The 'random SNPs' and 'stratified sampling' schemes differed in that the former effectively assumed that each SNP is typed in a different set of individuals, while the latter assumed that the same set of individuals is used for genotyping. We note that the latter scenario is typically the more appropriate one, although usually the impact on simulation outcomes is small. All three of these simulation types had 'matching' and 'no matching' versions. Confusingly, the type of 'matching' applied differed according to type. For 'random SNPs' simulations, valid inexact population-level matching was used. For 'differential SNPs' and 'stratified sampling', invalid inexact subpopulation matching was used (Zheng G; pers. comm.).

We note that in those studies above which investigated DC with appreciable PS (Zang et al. [11], Zhang et al. [19]), control of Type 1 error was only ever achieved in those simulations using invalid subpopulation allele frequency matching, and never in those simulations using valid population-level allele frequency matching.

\section{Methods}

Gorroochurn et al. [7] introduced several standardised scenarios of a dominant genetic model in two subpopulations (see their table 1). To investigate whether Type I error is properly controlled, we chose to investigate four of their scenarios involving PS but no true association ( $8,13,15,17$, used in their table 4). These cover a range of potential PS situations in decreasing order of severity (the left-hand column of our table 2 ). We used both their methods to estimate $\delta$, referred to here as the $\hat{\delta}_{2006}$ and $\hat{\delta}_{2007}$ methods of estimation.

First we replicated the findings of Gorroochurn et al. [7] for each scenario. Using their simulation technique, which matches allele frequencies within each subpopulation, we simulated 1 test SNP and 100 null SNPs and estimated $\delta$ using $\delta_{2006}$ and $\delta_{2007}$. This was repeated 10,000 times. We calculated Type 1 error under both methods, matching null SNP frequencies in controls to \pm 0.15 as suggested by Gorroochurn et al. [6, 7].

Next, we repeated this work with data simulated using the Balding-Nichols method [15]. In order to do this, we needed to sample values of $r_{i}$ from an appropriate beta distribution for each subpopulation within each simulation. We achieved this by averaging the scenario-specific $r_{1}$ and $r_{2}$ to get $r_{m u}$ (the populationaverage probability of having genotype $\mathrm{M}$ ), calculating $p_{m u}$ (the population-average allele frequency) and sampling $p_{1}^{\prime}$ and $p_{2}^{\prime}$ from $\operatorname{Beta}\left(\left(1-F_{S T}\right) / F_{S T} \times p_{m u},\left(1-F_{S T}\right) / F_{S T} \times\left(1-p_{m u}\right)\right)$ where $F_{S T}$ is the conventional parameter used to describe the level of genetic differentiation between populations. $F_{S T}$ was calculated from the basic PS parameters assumed in Gorroochurn et al.'s [6] simulations (using the values from their table 1). Then $r_{1}^{\prime}, r_{2}^{\prime}, s^{\prime}$ and $t^{\prime}$ were calculated, and finally the case-control genotypes were obtained using binomial sampling as before. Thus, the allele frequencies in subpopulations 1 and 2 were not known in advance, but should have the same mean and are subject to the same level of genetic drift assumed within the simulations of Gorroochurn et al. [6]. Delta was estimated using both $\hat{\delta}_{2006}$ and $\hat{\delta}_{2007}$ and Type 1 error calculated from 10,000 simulations as previously, with the same level of frequency matching in null SNPs. The right-hand columns of table 2 list the $r_{m u}$ and $F_{S T}$ used.

As a comparison, we also applied GC (both $\mathrm{GC}_{\mathrm{mu}}$ and GCF) using the same null SNPs. We used parameters settings very similar to those originally used by Gorroochurn [7] (sample size of 200 cases and 200 controls, tolerance levels of \pm 0.15 on null SNP genotype frequency matched on controls), but also briefly tested the robustness of our conclusions to these parameters.

\section{Results}

When using their proposed simulation algorithm, our simulations replicated the findings of Gorroochurn et al. $[6,7]$ for each scenario considered. The results for $\hat{\delta}_{2006}$ and $\hat{\delta}_{2007}$, and for Type 1 error are given in table 3 in descending order of 'true' $\delta$ and are very similar to those given in table 4, column 1 of Gorroochurn et al. [7]. We found similar values of 'recovered' $\delta$ to 'true' $\delta$ with only 
Table 2. Parameters for selected scenarios defined by Gorroochurn and adapted for use with the Balding-Nichols simulation method, in descending order of the degree of PS as measured by 'true' $\delta$

\begin{tabular}{|c|c|c|c|c|c|c|c|}
\hline \multirow[t]{2}{*}{ Scenario } & \multicolumn{5}{|c|}{ Gorroochurn } & \multicolumn{2}{|c|}{ Balding-Nichols } \\
\hline & 'True' $\delta$ & Pop & $\pi_{1}, \pi_{2}$ & $d_{1}, d_{2}$ & $r_{1}, r_{2}$ & $r_{m u}$ & $F_{S T}$ \\
\hline \multirow[t]{2}{*}{17} & \multirow[t]{2}{*}{2.003} & 1 & 0.2620 & 0.2502 & 0.4770 & \multirow[t]{2}{*}{0.3826} & \multirow[t]{2}{*}{0.018} \\
\hline & & 2 & 0.7380 & 0.03326 & 0.2882 & & \\
\hline \multirow[t]{2}{*}{15} & \multirow[t]{2}{*}{1.503} & 1 & 0.2620 & 0.2502 & 0.4770 & \multirow[t]{2}{*}{0.3826} & \multirow[t]{2}{*}{0.018} \\
\hline & & 2 & 0.7380 & 0.05855 & 0.2882 & & \\
\hline \multirow[t]{2}{*}{13} & \multirow[t]{2}{*}{1.003} & 1 & 0.5241 & 0.2818 & 0.4298 & \multirow[t]{2}{*}{0.2902} & \multirow[t]{2}{*}{0.050} \\
\hline & & 2 & 0.4759 & 0.1654 & 0.1505 & & \\
\hline \multirow[t]{2}{*}{8} & \multirow[t]{2}{*}{0.498} & 1 & 0.7648 & 0.1908 & 0.5614 & \multirow[t]{2}{*}{0.3549} & \multirow[t]{2}{*}{0.061} \\
\hline & & 2 & 0.2352 & 0.1416 & 0.1484 & & \\
\hline
\end{tabular}

Table 3. Estimates of $\delta$ and Type 1 error using Gorroochurn's original simulation technique, the Balding-Nichols simulation technique

\begin{tabular}{|c|c|c|c|c|}
\hline \multirow{2}{*}{$\begin{array}{l}\text { Simulation } \\
\text { technique }\end{array}$} & \multicolumn{4}{|c|}{ Scenario } \\
\hline & 17 & 15 & 13 & 8 \\
\hline \multicolumn{5}{|l|}{$\hat{\delta}_{2006}$} \\
\hline Gorr & 2.003 & 1.503 & 1.003 & 0.497 \\
\hline B-N & 0.0182 & 0.0087 & 0.0095 & 0.0007 \\
\hline \multicolumn{5}{|l|}{$\hat{\delta}_{2007}$} \\
\hline Gorr & 2.014 & 1.511 & 1.008 & 0.500 \\
\hline B-N & 0.0022 & 0.0093 & -0.0095 & -0.0180 \\
\hline \multicolumn{5}{|l|}{ Type 1 (UN) } \\
\hline Gorr & 0.507 & 0.325 & 0.171 & 0.083 \\
\hline B-N & 0.228 & 0.161 & 0.107 & 0.0624 \\
\hline \multicolumn{5}{|c|}{ Type $1\left(\hat{\delta}_{2006}\right)$} \\
\hline Gorr & 0.0516 & 0.0515 & 0.0480 & 0.0520 \\
\hline $\mathrm{B}-\mathrm{N}$ & 0.197 & 0.124 & 0.104 & 0.0592 \\
\hline \multicolumn{5}{|c|}{ Type $1\left(\hat{\delta}_{2007}\right)$} \\
\hline Gorr & 0.0517 & 0.0557 & 0.0513 & 0.0509 \\
\hline $\mathrm{B}-\mathrm{N}$ & 0.185 & 0.125 & 0.105 & 0.0636 \\
\hline \multicolumn{5}{|c|}{ Type $1\left(\mathrm{GC}_{\mathrm{mu}}\right)$} \\
\hline Gorr & 0.0080 & 0.0234 & 0.0404 & 0.0536 \\
\hline $\mathrm{B}-\mathrm{N}$ & 0.0577 & 0.0554 & 0.0545 & 0.0505 \\
\hline \multicolumn{5}{|c|}{ Type 1 (GCF) } \\
\hline Gorr & 0.0072 & 0.0215 & 0.0383 & 0.0510 \\
\hline $\mathrm{B}-\mathrm{N}$ & 0.0550 & 0.0528 & 0.0505 & 0.0489 \\
\hline
\end{tabular}

Type 1 errors for the uncorrected statistics (UN) and for $\mathrm{GC}_{\mathrm{mu}}$ and GCF are also given. minor differences between $\hat{\delta}_{2007}$ and $\hat{\delta}_{2007}$, and the empirical Type 1 errors for all methods are similar to those found by Gorroochurn et al. [6, 7]. We therefore confirm that under the simulation conditions used by Gorroochurn et al. $[6,7]$ the DC method appears to appropriately correct Type 1 errors inflated through PS. Likewise, we confirm that the reduced variability present under these simulation conditions results in a severe over-correction under GC, with highly conservative Type 1 errors and by implication reduced power.

We then repeated the scenarios, simulating under the Balding-Nichols model (table 3). Under these conditions, $\delta$ is poorly estimated by both $\hat{\delta}_{2007}$ and $\hat{\delta}_{2007}$ and consequently DC does not correct empirical Type 1 error. In contrast, both GC methods now have approximately the right empirical Type 1 error at the 5\% level, consistent with previous results [8]. It is noticeable that the uncorrected Type 1 error is lower than for the Gorroochurn simulation method, indicating that the level of PS under the Balding-Nichols model is much lower than originally found by Gorroochurn, as a side effect of simulating null allele frequencies from a distribution with greater variance but under more valid population genetic assumptions.

We repeated the simulations under a range of sample sizes for the association study and tolerance levels on genotype frequency matching for the test and null SNPs (work not shown). Our conclusions are generally robust to these variations, although we note that under higher levels of PS than that described by scenario 17 the DC method can sometimes be anti-conservative rather than conservative. 


\section{Discussion}

In this article we have argued that the simulation technique implemented by Gorroochurn in 2006 and 2007 is flawed. Neither artificial processes (i.e. selection by the researcher, based on limited null SNPs) nor natural population genetic processes can reasonably result in the subpopulation allele frequency matching assumed by their simulations. When a more appropriate simulation technique is applied (the Balding-Nichols method), the noncentrality parameter $\delta$ is not well estimated using either of the two DC methods described $\left(\delta_{2006}\right.$ or $\left.\delta_{2007}\right)$, and the result is that very little correction is made for Type 1 error inflation caused by PS. We investigated DC only under the dominant genetic model, in keeping with most of the simulations performed by Gorroochurn and colleagues. However, we note that a similar difference in the results from subpopulation versus population-level allele frequency matching can also be found in Zang et al. [11] and Zhang et al. [19] for DC under the additive genetic model. Furthermore, as we review in our section on distributional theory, estimation of the $\delta$ parameter required by DC is fundamentally predicated on the idea that subpopulation allele frequency matching is possible. Without this assumption it is only possible to estimate $\delta^{2}$, which is the rationale behind GC. We conclude that DC is incapable of correcting for PS in genetic association studies and should not be used. In contrast, our simulations confirm that GC is an appropriate method of correction across a range of PS levels.

The Balding-Nichols model is not the only possible population genetic model. An important question is whether there are any plausible population genetic models which might result in a tendency for null and target SNP subpopulation allele frequencies to covary, as required by DC. One could consider, for example, a population splitting model where some subpopulations are more closely related than others because they split from a more recent ancestral population. This would break the assumption that the allele frequencies of a single SNP are independent and identically distributed (i.i.d.) among subpopulations, which is part of the Balding-Nichols model. However, even this more complex model would remain i.i.d., conditional on subpopulation, among unlinked null SNPs. Indeed, taking any one ancestral population as a starting point, the randomness of genetic drift should ensure that it is impossible to predict the direction of drift in one SNP by observing what happens in another unlinked one. The same principle would also apply to admixture models involving migration between subpop- ulations and to isolation-by-distance models where no discrete subpopulations exist. The only force that provides predictable directionality is selection, but for this to apply would require the same selective pressure acting on both test SNP and all null SNPs (violating the definition of a null SNP), and furthermore one would need to have prior knowledge of which allele was selectively advantageous for each SNP.

We conclude that even a rough matching of withinsubpopulation allele frequencies of the type required by DC is infeasible for any plausible population genetic model governing the allele frequency distribution of test and null SNPs. Under reasonable population genetic conditions, DC fails. However, we note that there may be other reasons for performing allele frequency matching averaged over all subpopulations, as advocated by Gorroochurn et al. [6, 7]. Reich \& Goldstein [12] found that allele frequency matching of this kind improved the power of GC in their simulations for cases where the minor allele frequency of the test SNP was less than 0.15. Devlin et al. [10] noted that $F_{S T}$ may vary with minor allele frequency because younger mutations tend to be rarer, which would also motivate matching. Finally, Zheng et al. [17] noted that if one wishes to employ $\chi^{2}$ tests under the dominant or recessive genetic model, then allele frequency matching is required because $\lambda$ depends on mean allele frequency.

We have also identified several studies [11, 17-19] which apply similarly flawed simulations involving subpopulation allele frequency matching. We have not conducted an in-depth investigation of the impact of this on the conclusions reached in these studies. We would argue, however, that all results and conclusions that depend solely on these flawed simulations should be treated as invalid. By the same token, we would argue that all theoretical derivations, as well as all conclusions based on simulations not containing this flaw (as many of the studies also contain these), should in principle still be considered valid.

Our findings raise some general issues regarding the correct use of simulations in studies of genetic association. Firstly, it is imperative that these simulations are based on sound population genetic principles, as genetic association studies by their very nature rely on population-based sampling. Secondly, it is important that simulation procedures are described with enough detail to allow complete construction of the algorithm used. In some cases, we believe this is not given appropriate priority. Finally, terms should be carefully defined in order to avoid confusion. As we note earlier, the term 'allele frequency 
matching' has multiple different possible meanings which has contributed to keeping hidden the problem of subpopulation allele frequency matching.

PS remains a challenge in genetic association studies, but there are now are a wide range of statistically valid methods available for estimating and correcting for PS, including GC, structured association, and principal components analysis. Our research demonstrates these methods should be used in preference to DC, which fails to correct test statistics effectively.

\section{Acknowledgements}

We would like to thank Gang Zheng and two anonymous referees for their helpful comments and discussions.

\section{References}

1 Tiwari HK, Barnholtz-Sloan J, Wineinger N, Padilla MA, Vaughan LK, Allison DB: Review and evaluation of methods correcting for population stratification with a focus on underlying statistical principles. Hum Hered 2008;66:67-86.

22 Devlin B, Roeder K: Genomic control for association studies. Biometrics 1999;55:9971004.

3 Pritchard JK, Stephens M, Donnelly P: Inference of population structure using multilocus genotype data. Genetics 2000;155:945959.

-4 Price AL, Patterson NJ, Plenge RM, Weinblatt ME, Shadick NA, Reich D: Principal components analysis corrects for stratification in genome-wide association studies. Nat Genet 2006;38:904-909.

5 Setakis E, Stirnadel H, Balding DJ: Logistic regression protects against population structure in genetic association studies. Genome Res 2006;16:290-296.

- 6 Gorroochurn P, Heiman GA, Hodge SE, Greenberg DA: Centralizing the non-central chi-square: A new method to correct for population stratification in genetic case-control association studies. Genet Epidemiol 2006; 30:277-289.
7 Gorroochurn P, Hodge SE, Heiman GA, Greenberg DA: A unified approach for quantifying, testing and correcting population stratification in case-control association studies. Hum Hered 2007;64:149-159.

8 Dadd T, Weale ME, Lewis CM: A critical evaluation of genomic control methods for genetic association studies. Genet Epidemiol 2009;33:290-298.

-9 The Wellcome Trust Case-Control Consortium: Genome-wide association study of 14,000 cases of seven common diseases and 3,000 shared controls. Nature 2007;447:661678.

10 Devlin B, Roeder K, Bacanu SA: Unbiased methods for population-based association studies. Genet Epidemiol 2001;21:273-284.

11 Zang Y, Zhang H, Yang Y, Zheng G: Robust genomic control and robust delta centralization tests for case-control association studies. Hum Hered 2007;63:187-195.

12 Reich DE, Goldstein DB: Detecting association in a case-control study while correcting for population stratification. Genet Epidemiol 2001;20:4-16.

13 Devlin B, Bacanu SA, Roeder K: Genomic control to the extreme. Nat Genet 2004;36: 1129-1130.

14 Sasieni PD: From genotypes to genes: Doubling the sample size. Biometrics 1997;53: 1253-1261.
$>15$ Balding DJ, Nichols RA: A method for quantifying differentiation between populations at multi-allelic loci and its implications for investigating identity and paternity. Genetica 1995;96:3-12.

16 Zheng G, Friedlin B, Gastwirth JL: Robust genomic control for association studies. Am J Hum Genet 2006;78:350-356.

17 Zheng G, Freidlin B, Li Z, Gastwirth JL: Genomic control for association studies under various genetic models. Biometrics 2005;61: 186-192.

18 Zheng G, Li Z, Gail MH, Gastwirth JL: Impact of population substructure on trend tests for genetic case-control association studies. Biometrics 2009 (Epub ahead of print).

19 Zhang H, Li Q, Zang Y, Yang YN, Zheng G: Centralized genomic control: A simple approach correcting for population structures in case-control association studies; in Balakrishnan N (ed): Methods and Applications of Statistics in the Life and Health Sciences, New York: Wiley, 2009, pp 81-93.

20 Astle W, Balding DJ: Population structure and cryptic relatedness in genetic association studies. Stat Sci, in press. 


\section{Erratum}

In the article 'Delta-centralization fails to control for population stratification in genetic association studies' by Dadd et al. [Hum Hered 2010;69:285-294] the following errors appeared:

1) Reference 10 should refer to: Devlin B, Roeder K and Wasserman L: Genomic control, a new approach to genetic-based association studies. Theor Popul Biol 2001;60: $155-166$.

2) On page 288, the equation for the bias parameter $\delta_{T}$ should be:

$$
\delta_{T}=\sum_{j=1}^{K} p_{T j} f_{j} / s
$$

Also the term ' $s$ ' needs to be defined as: 'where $s$ is an estimate of the standard deviation of the difference in allele frequency between cases and controls.'

3) On page 288 , the equation for $\operatorname{Var}\left[\delta_{T}\right]$ should be:

$$
\operatorname{Var}\left[\delta_{i}\right]=F_{S T} p_{i}\left(1-p_{i}\right) \sum_{j=1}^{K} f_{j}^{2} / s^{2}
$$

4) On page 288, the equation for $\eta^{2}$ should be:

$$
\eta^{2}=n F_{S T} \sum_{j=1}^{K} f_{j}^{2}
$$

5) On page 288, the expression

$$
\sum_{j=1}^{K} f_{j}
$$

which appears half way down the right hand side should be:

$$
\sum_{j=1}^{K} f_{j}^{2}
$$

